\title{
A FIXED-POINT THEOREM FOR TREES 1
}

\author{
A. D. WALLACE
}

By a tree we mean a compact (= bicompact) Hausdorff space which is acyclic in the sense that

(i) if $\mathfrak{U}$ is a f.o.c. (= finite open covering) of a tree $T$ then there is a f.o.c. $\mathfrak{B} \subset \mathfrak{U}$ such that the nerve $N(\mathfrak{B})$ is a combinatorial tree,

and which is locally connected in the sense that

(ii) if $\mathfrak{U}$ is a f.o.c. of $T$ then there is a f.o.c. $\mathfrak{B} \subset \mathfrak{U}$ whose vertices are connected sets.

It may be shown [3] that an acyclic continuous curve in the usual sense is a tree in our terminology. If $q$ is a mapping which assigns to each point $t$ of a topological space a set $q t$ in a topological space, then we say that $q$ is continuous provided that for each $t$ and each neighborhood $U$ of $q t$ we can find an open set $V$ containing $t$ such that if $t^{\prime}$ is in $V$ then $q t^{\prime}$ is in $U$. Our present purpose is to establish the following result:

(A) Let $T$ be a tree and let $q$ be a continuous point-to-set mapping which assigns to each point t a continuum qt in $T$. Then there is $a t_{0} \in T$ such that $t_{0} \in q t_{0}$.

The proof (which is divided into several lemmas) uses strongly a technique introduced by $\mathrm{H}$. Hopf [1]. However the present note has been made self-contained.

$\left(\mathrm{A}_{1}\right)$ The intersection of two continua of $T$ is again a continuum.

Proof. Let $B_{1}, B_{2}$ be two continua such that $B_{1} \cdot B_{2}=C_{1}+C_{2}$ where the $C_{i}$ are disjoint and closed. We can find disjoint open sets $D_{i} \supset C_{i}$. Let $t \in T-B_{1} \cdot B_{2}$. We can then find an open set $V_{t}$ containing $t$ and which does not meet both $B_{1}$ and $B_{2}$. The sets $D_{i}$ together with the sets $V_{t}$ can be reduced to a f.o.c. $\mathfrak{U}$ of $T$. Let $\mathfrak{B} \subset \mathfrak{U}$ be the f.o.c. described in (i). Let $\mathfrak{B}_{i}$ be those vertices of $\mathfrak{B}$ on $B_{i}$. It is easy to see that $N\left(\mathfrak{B}_{i}\right)$ is connected. If $c_{j} \in C_{j}$ we can find a chain of 1-cells $E_{i}$ in $N\left(\mathfrak{B}_{i}\right)$ whose first vertex contains $c_{1}$ and whose last vertex contains $c_{2}$. Now we cannot have $E_{i} \subset D_{1}+D_{2}$ and $E_{i}$ contains a vertex which is not on $B_{j}$. Hence $E_{1} \neq E_{2}$ and so $N(\mathfrak{B})$ is not a tree. This contradiction completes the proof.

\footnotetext{
${ }^{1}$ Presented to the Society, May 3, 1941.
} 
$\left(\mathrm{A}_{2}\right)$ Any f.o.c. $\mathfrak{U}$ of $T$ contains a f.c.c. $\mathfrak{F} \subset \mathfrak{U}$ so that each $F_{i} \in \mathfrak{F}$ is connected and further $N(\mathfrak{F})$ is a combinatorial tree.

Proof. We can find a f.o.c. $\mathfrak{B} \subset \mathfrak{U}$ such that $N(\mathfrak{B})$ is a tree. By a lemma due to Čech $\left[5\right.$, p. 180] we can find a f.c.c. $\mathfrak{F}^{\prime} \subset \mathfrak{B}$ such that $\mathfrak{F}^{\prime}$ and $\mathfrak{B}$ are combinatorially isomorphic. Let $\Re_{i}$ be the f.o.c. $\left(V_{i}, T-F_{i}^{\prime}\right)$. Using (ii) it is easy to see that there is a f.o.c. $\mathfrak{W}$ such that each $W_{i}$ is connected and $\overline{\mathfrak{W}} \subset \Re_{i}$, for each $i$. Let $i$ be fixed. If $W_{j}$ meets $F_{i}^{\prime}$ then so does $\bar{W}_{j}$ and so is contained in $V_{i}$. Let $Q_{i}$ be the union of all such $W_{j}$. Then the closure of this set has a component-wise decomposition, say $\bar{Q}_{i}=F_{i 1}+F_{i 2}+\cdots+F_{i s_{i}}$. Let $\mathfrak{F}$ be the f.c.c. $\left\{F_{i j}\right\}$. It is clear that the elements of $\mathfrak{F}$ are connected and it is not hard to show that $\operatorname{dim} \mathfrak{F} \leqq 1$, that is, at most two elements of $\mathfrak{F}$ have a non-null intersection. If we have a chain

$$
F_{i_{1} j_{1}}, F_{i_{2} j_{2}}, \cdots, F_{i_{r} j_{r}}, F_{i_{1} j_{1}},
$$$$
r>2 \text {, }
$$

such that each set meets the following but such that there are no other intersections, then the sets $F_{i_{1} j_{1}}$ and $\sum_{s>1} F_{i_{s} j_{s}}$ are connected and therefore by $\left(\mathrm{A}_{1}\right)$ so is their meet, the set $F_{i_{1} j_{1}} \cdot F_{i_{2} j_{2}}+F_{i_{1} j_{1}} \cdot F_{i_{r} j_{r}}$. But then we would have $F_{i_{1} j_{1}} \cdot F_{i_{2} j_{2}} \cdot F_{i_{r} j_{r}} \neq 0$, a contradiction. It follows that $N(\mathfrak{F})$ is a tree.

(B) Let $q$ be a mapping which assigns to each continuum $K$ in $T$ a continuum $q K$ in $T$ such that if $K_{1} \subset K_{2}$, then $q K_{1} \subset q K_{2}$. If $\mathfrak{F}=\left\{F_{i}\right\}$ is a f.c.c. with connected sets such that $N(\mathfrak{F})$ is a tree then there is an $F_{i}$ for which $F_{i} \cdot q F_{i} \neq 0$.

Proof. Let $N=N(\mathfrak{F})$ and suppose that the vertices of $N$ are $e_{i}$. To each $i$ we assign an $i^{\prime}$ so that $F_{i}$, meets $q F_{i}$. We then have a mapping $e_{i} \rightarrow e_{i}$, and since $N$ is a tree it follows at once by a result due to Hopf $[1$, Lemma $\gamma]$ that we can find an edge $e_{m} e_{n}$ which is contained in the chain joining $e_{m}$, to $e_{n}^{\prime}{ }^{2}$ We show that $F_{k} \cdot q F_{k} \neq 0, k=m$, or $n$. We have $F_{m} \cdot F_{n} \neq 0$ and by construction $F_{m} \cdot q F_{m} \neq 0 \neq F_{n^{\prime}} \cdot q F_{n}$. Further

$$
F_{m^{\prime}}, F_{i}, \cdots, F_{m}, F_{n}, F_{j}, \cdots, F_{n^{\prime}}
$$

is a simple chain of sets. Of course it may happen that $F_{n}$ precedes $F_{m}$ in $\left(^{*}\right)$ but this is of no importance. Let $X$ be the union of all the sets in (*) from $F_{m}$, up to and including $F_{m}$. Let $Y$ be similarly defined for the other part of $\left(^{*}\right)$. Then $X$ and $Y$ are continua with $X \cdot Y=F_{m} \cdot F_{n}$.

${ }^{2} \mathrm{I}$ am indebted to Professor S. Lefschetz for the remark that $e_{i} \rightarrow e_{i}$ generates a chain-mapping (that is, a mapping permutable with the boundary operator) if we define for the image of $e_{m} e_{n}$ the chain joining $e_{m}$ to $e_{n}$. Since $N$ is acyclic it follows at once that there is a fixed element. This may replace the result of Hopf. 
Also $F_{m}+F_{n}$ is a continuum and so is $Z=q F_{m}+q F_{n}$. Clearly $Z$ meets the end-vertices of $\left(^{*}\right)$. By $\left(\mathrm{A}_{1}\right) Z \cdot(X+Y)$ is a continuum. Hence $Z \cdot X \cdot Y$ is not null. Thus $F_{m} \cdot F_{n} \cdot\left(q F_{m}+q F_{n}\right) \neq 0$ and this completes the proof of $(B)$.

It is not hard to see that if $q$ is a mapping of the type described in (A) then $q$ satisfies the conditions in (B) if we define $q K=\sum q t, t \in K$, for each continuum $K$ of $T$. The proof is quite similar to those for analogous results concerning single-valued mappings.

We now turn to a proof of (A). Suppose that no $t$ is in $q t$. We can find a neighborhood $R_{t}$ of $t$ so that $\bar{R}_{t}$ does not meet $q t$. Let $V_{t}=T-\bar{R}_{t}$. Since $q t \subset V_{t}$ we can find a neighborhood $S_{t}$ of $t$ so that $t^{\prime} \in S_{t}$ implies $q t^{\prime} \subset V_{t}$. Let $U_{t}$ be the meet of $R_{t}$ and $S_{t}$. We cover $T$ by a finite subcollection $\left\{U_{i}\right\}=\left\{U_{t_{i}}\right\}$ of the sets $U_{t}$. We can find a refinement $\mathfrak{F}$ of $\mathfrak{U}=\left\{U_{i}\right\}$ which satisfies the conditions in (B) in consequence of $\left(\mathrm{A}_{2}\right)$. By $(\mathrm{B})$ we can find a set $F$ in $\mathfrak{F}$ so that $F$ meets $q F$. In other words we find a $t$ in $F$ such that $F$ meets $q t$. Now $F$ is in some $U_{i}$ and hence $q t$ is in the corresponding $V_{i}$. But since $F$ does not meet the set $V_{i}$ it cannot meet $q t$. This contradiction completes the proof.

A continuous transformation $f M=N$ is said to be free (Hopf [1]) provided there is a continuous transformation $g M \subset M$ such that $f g x \neq f x$ for each $x \in M$. The transformation $f$ is monotone if the set $f^{-1} y$ is connected for each $y \in N$.

(C) No continuum admits a free monotone transformation onto a tree.

Proof. Let $f M=T$ be monotone and $g M \subset M$ be continuous. For each $t \in T$ we set $q t=f g f^{-1} t$. It is not hard to see that $q$ is continuous and hence we may apply (A). But from $t \in q t$ it follows at once that there is an $x \in M$ with $f g x=f x$.

The transformations $f M \subset N$ and $g M \subset N$ have a coincidence (Lefschetz [2]) if there is an $x \in M$ with $f x=g x$. As in (C) we may show that

(D) A monotone transformation $f M=T$ of a continuum onto a tree admits a coincidence with any continuous transformation $g M \subset T$.

Remarks. The result (A) is usually called the Scherrer fixed-point theorem when $q$ is single-valued and $T$ is an acyclic continuous curve. For a list of papers concerning it see Hopf [1]. Corollary (C) will be found in [3]. The result (A) was found while constructing a proof of (D). Finally (A) is analogous to a result of S. Kakutani [4] who has shown that if $S$ is an $n$-simplex and to each $s \in S$ we assign continuously a closed convex set $q s$ then there is an $s_{0} \in q s_{0}$. 


\section{BIBLIOGRAPHY}

1. H. Hopf, Fundamenta Mathematicae, vol. 28 (1937), p. 31.

2. S. Lefschetz, Topology, American Mathematical Society Colloquium Publications, vol. 12, New York, 1930.

3. A. D. Wallace, Duke Mathematical Journal, vol. 6 (1940), p. 31.

4. S. Kakutani, A generalization of Brouwer's fixed-point theorem, to appear in the Duke Mathematical Journal.

5. E. Cech, Fundamenta Mathematicae, vol. 25 (1932), p. 149.

Princeton University

\section{ON THE DEFINITION OF CONTACT TRANSFORMATIONS}

\section{ALEXANDER OSTROWSKI}

If $z$ is a function of $x_{1}, \cdots, x_{n}$ and $p_{\nu}=\partial z / \partial x_{\nu}, \nu=1, \cdots, n$, a contact transformation in the space of $z, x_{1}, \cdots, x_{n}$, is defined by a set of $n+1$ equations

$$
Z=Z\left(z, x_{\mu}, p_{\mu}\right), \quad X_{\nu}=X_{\nu}\left(z, x_{\mu}, p_{\mu}\right), \quad \nu=1, \cdots, n,
$$

such that firstly in calculating the $n$ derivatives

$$
P_{\nu}=\frac{\partial Z}{\partial X_{\nu}}, \quad \quad \nu=1, \cdots, n,
$$

the expressions for the $P_{\nu}$ are given by a set of $n$ equations

$$
P_{\nu}=P_{\nu}\left(z, x_{\mu}, p_{\mu}\right), \quad \nu=1, \cdots, n,
$$

in which the derivatives of the $p_{\mu}$ fall out; and secondly the equations (a) and (b) can be resolved with respect to $z, x_{\mu}, p_{\mu}$ :

$$
\begin{array}{cc}
z=z\left(Z, X_{\mu}, P_{\mu}\right), \quad x_{\nu}=x_{\nu}\left(Z, X_{\mu}, P_{\mu}\right), & \nu=1, \cdots, n, \\
p_{\nu}=p_{\nu}\left(Z, X_{\mu}, P_{\mu}\right), & \nu=1, \cdots, n .
\end{array}
$$

These two postulates are equivalent with the hypothesis that the $2 n+1$ equations (a), (b) form a transformation between the two spaces of the sets of $2 n+1$ independent variables $\left(z, x_{\nu}, p_{\nu}\right),\left(Z, X_{\nu}, P_{\nu}\right)$ satisfying the Pfaffian condition

$$
d Z-\sum_{\nu=1}^{n} P_{\nu} d X_{\nu}=\rho\left(d z-\sum_{\nu=1}^{n} p_{\nu} d x_{\nu}\right), \quad \rho \neq 0 .
$$

